\title{
Domination of Fuzzy Incidence Graphs with the Algorithm and Application for the Selection of a Medical Lab
}

\author{
Irfan Nazeer $\mathbb{D}^{1},{ }^{1}$ Tabasam Rashid $\mathbb{D}^{1},{ }^{1}$ and Juan Luis Garcia Guirao $\mathbb{i D}^{2,3}$ \\ ${ }^{1}$ University of Management and Technology, Lahore 54770, Pakistan \\ ${ }^{2}$ Departamento de Matematica Aplicada y Estadistica, Universidad Politecnica de Cartagena, Cartagena, Spain \\ ${ }^{3}$ Nonlinear Analysis and Applied Mathematics (NAAM)-Research Group, Department of Mathematics, Faculty of Science, \\ King Abdulaziz University, P.O. Box 80203, Jeddah 21589, Saudi Arabia \\ Correspondence should be addressed to Juan Luis Garcia Guirao; jlgarcia@kau.edu.sa
}

Received 7 October 2020; Revised 9 February 2021; Accepted 19 April 2021; Published 11 May 2021

Academic Editor: Mahmoud Mesbah

Copyright (c) 2021 Irfan Nazeer et al. This is an open access article distributed under the Creative Commons Attribution License, which permits unrestricted use, distribution, and reproduction in any medium, provided the original work is properly cited.

\begin{abstract}
Fuzzy graphs (FGs), broadly known as fuzzy incidence graphs (FIGs), have been recognized as being an effective tool to tackle realworld problems in which vague data and information are essential. Dominating sets (DSs) have multiple applications in diverse areas of life. In wireless networking, DSs are being used to find efficient routes with ad hoc mobile networks. In this paper, we extend the concept of domination of FGs to the FIGs and show some of their important properties. We propose the idea of order, size, and domination in FIGs. Two types of domination, namely, strong fuzzy incidence domination and weak fuzzy incidence domination, for FIGs are discussed. A relationship between strong fuzzy incidence domination and weak fuzzy incidence domination for complete fuzzy incidence graphs (CFIGs) is also introduced. An algorithm to find a fuzzy incidence dominating set (FIDS) and a fuzzy incidence domination number (FIDN) is discussed. Finally, an application of fuzzy incidence domination (FID) is provided to choose the best medical lab among different labs for the conduction of tests for the coronavirus.
\end{abstract}

\section{Introduction}

The graph concept stands as one of the most dominant and widely employed tools for multiple real-world problem representation, modeling, and analysis. To indicate the objects and the relations between them, the graph vertices or nodes and edges or arcs are applied, respectively. In graphs, the notion of domination first took place in the game of chess during the 1850s. In Europe, lovers of chess thought carefully about the complication of fixing the fewer numbers of queens that can be laid down on a chessboard so that all the squares are engaged by a queen. The idea of domination began with Claude Berge and Ore [1]. Domination in graphs has plenty of applications in a variety of areas. Concepts from DSs appear in problems involving finding sets of representatives in monitoring communication networks. Hosamani et al. [2] proposed an idea of c-dominating energy in graphs and obtained various properties of c-dominating energy. Harisha et al. [3] discussed degree sequence and join operations in graphs. Mathew et al. [4] introduced vertex rough graphs and the membership function of vertex rough graphs. For more detailed study on graphs, we may suggest to the reader [5-10].

When there are uncertainty and ambiguity among relationships of different objects, the graphs are not enough. This lack in graphs led Zadeh [11] to propose an idea of fuzzy sets (FSs). FSs are beneficial when there is inconclusiveness among different objects. After the landmark work of Zadeh on FSs, Rosenfeld [12] gave the notion of FGs. The study of FGs opens a new door for many researchers to participate in this field such as Gani and Ahamed [13] examined the properties of different kinds of degree, order, and size of FGs and compared the relationship between degree, order, and size of FGs. Akram [14] presented an idea of bipolar FGs and investigated their various properties. Bhattacharya [15] gave the notion of eccentricity and center of FGs. Rashmanlou and Jun [16] introduced complete interval-valued FGs. Somasundaram and Somasundaram [17] presented an outstanding idea of domination in FGs. Somasundaram [18] discussed various types of products such as union, join, 
composition, and Cartesian product and their domination parameters for FGs. Gani and Ahamed [19] introduced strong and weak domination in FGs. Manjusha and Sunitha $[20,21]$ suggested the idea of total domination using strong arcs and strong domination in FGs, respectively. Ponnappan [22] et al. proposed the novel idea of edge domination, total edge domination, the edge domination number, and the total edge domination number for certain classes of FGs. Dharmalingam and Rani [23] proposed an idea of equitable domination in FGs. Dharmalingam and Nithya [24, 25] discussed the concept of excellent domination and very excellent domination in FGs. Wang et al. [26, 27] addressed the $H_{\infty}$ control and filtering problems for Markov jump singularity perturbed systems approximated by Takagi-Sugeno fuzzy models and stability analysis on singularity perturbed descriptor systems. For some other significant and constructive works on FGs, we may refer to [28-34].

There is a drawback of FGs because they are unspeakable to give any clue of the effect of a vertex on an edge. This deficiency in FGs opens a new door to introduce the idea of FIGs. FIGs are more impressive than FGs because in FGs, the membership value (MSV) of an edge $x y$ is always equal to the MSV of an edge $y x$, but in FIGs, the MSV of $(x, x y)$ may or may not be equal to $(y, x y)$. For example, if nodes represent various residence colonies and arcs express roads connecting these colonies, we can have an FG expressing the extent of traffic from one colony to another. The colony with a large number of colonists will have a large number of ramps in the colony. So, if $x$ and $y$ are two colonies and $x y$ is a road connecting them, then $(x, x y)$ could indicate the ramp system from the road $x y$ to the colony $x$. For the unweighted graph, both $x$ and $y$ will have an impact of 1 on $x y$. For the directed graph, the effect of $x$ on $x y$ represented by $(x, x y)$ is 1 , whereas $(y, x y)$ is 0 . This concept is generalized through FIGs. Dinesh [35] gave the notion of FIGs. Mathew and Mordeson [36] discussed connectivity concepts and other structural properties of FIGs. Mordeson and Mathew [37] developed the idea of fuzzy end nodes and fuzzy incidence cut vertices in FIGs. Mordeson et al. [38] proposed an innovative idea of vague FIGs and their eccentricity. They applied the vague FIGs to problems involving human trafficking and illegal immigration. For a more detailed and comprehensive study on FIGs, we may suggest to the reader $[39,40]$.

The main motivation of our work is that the degree, order, size, and domination in graphs and FGs are discussed by many researchers, but no one has explored these ideas for FIGs. That is why we propose these ideas for FIGs. Through these ideas, we can study different characteristics of FIGs. In graphs and FGs, if vertex $u$ dominates vertex $v$, then $v$ also dominates $u$, but in FIGs, if vertex $u$ dominates $v$, then it is not necessary that $v$ also dominates $u$. For example, five different countries $C_{1}, C_{2}, C_{3}, C_{4}$, and $C_{5}$ have friendship with each other. If $C_{1}$ dominates $C_{5}$ or some other countries concerning the education system, then it is not necessary that $C_{5}$ or other countries also dominate $C_{1}$. This encourages us to introduce the concept of domination for FIGs.

The remainder of this article is formulated as follows. Section 2 provides some preliminary results. Order, size, and their relationship in FIGs are discussed in Section 3. FID and complement of FIGs are given in Section 4. Section 5 demonstrates the types of domination such as strong FID and weak FID. A relationship between strong and weak FID for CFIGs is also discussed in the same section. Section 6 provides an application of FID in the selection of the best medical lab among different medical labs. A comparative analysis of our study with a previous study is discussed in Section 7. Conclusions and prospects are elaborated in Section 8.

In this paper, minimum and maximum operators are represented by $\wedge$ or min and $\vee$ or max, respectively. Some of the basic definitions and results are given in the following to comprehend the remaining contents of the article. These definitions are taken from $[11,17]$.

\section{Preliminaries}

A mapping $\mu: Z \longrightarrow[0,1]$ is called a fuzzy subset (FS) of Z. An FG with $M$ as the underlying set is a pair $G=(\varphi, \chi)$, where $\varphi: M \longrightarrow[0,1]$ is FS, $\chi: M \times M \longrightarrow[0,1]$ is a fuzzy relation on the FS $\varphi$ such that $\chi(u, v) \leq \varphi(u) \wedge \varphi(v)$ for all $u, v \in M$, and $M$ is a finite set. $O(G)=\sum_{u \in M} \varphi(u)$ is called the order of a graph, and $S(G)=\sum_{u, v \in M} \chi(u, v)$ is called the size of $G$. An FG is complete if $\chi(u, v)=\varphi(u) \wedge \varphi(v)$ for all $u, v \in V$. A complete FG is represented by $K_{\varphi}$. In an FG, if $\chi($ uv $)=\varphi(u) \wedge \varphi(v)$, then $u$ dominates $v$ and $v$ dominates $u$. A subset $M$ of $V$ is named as DS in $G$ if for each $v$ which does not belong to $M, \exists u \in M$ such that $u$ dominates $v$. The domination number (DN) of $G$ is the lowest cardinality of a DS among all DSs in $G$. The DN of $G$ is expressed by $\gamma(G)$ or $\gamma$. A DS $M$ of an FG is minimal DS if no proper subset of $M$ is a DS of an FG. $N(u)=\{v \in V \mid \chi(\mathrm{uv})=\varphi(u) \wedge \varphi(v)\}$ is said to be the neighborhood of $u$, and $N[u] \cup\{u\}$ is called the close neighborhood of $u$. For an FG, we can generalize a degree of a node in distinct methods. The sum of the weights of the EEs incident at node $n$ is said to be the effective degree (ED) of node $n$. It is shown by $\mathrm{d} E(n)$. $\delta_{E}(G)=\wedge\{\mathrm{d} E(n) \mid n \in V\}$ shows the lowest ED, and $\Delta_{E}(G)=$ $\vee\{\mathrm{d} E(n) \mid n \in V\}$ represents the highest ED. The neighborhood degree of $n$ is defined by $\sum_{m \in N(n)} \varphi(m)$, and it is represented by $\mathrm{d} N(n) . \delta_{N}(G)$ and $\Delta_{N}(G)$ express the lowest and highest neighborhood degree, respectively. In an FG, a node $m$ is called an isolated node if $\chi(\mathrm{mn})<\varphi(m) \wedge \varphi(n)$ for all $\mathrm{mn} \in \chi$.

Definition 1 (see [36]). Let $G$ be a simple graph having node set $V$ and edge set $E$. Then, $G=(V, E, I)$ is named as an incidence graph (IG), where $I \subseteq V \times E$. Figure 1 represents an IG. $(u, u v)$ is said to be an incidence pair (IP) if $(u, u v) \in$ IG.

Definition 2 (see [36]). Let $G$ be a graph with a vertex set $V$ and an edge set $E$ and $\varphi$ and $\chi$ be FSs of a vertex set and an edge set, respectively. Consider $\psi$ to be a FS of $V \times E$. If $\psi(g, h) \leq \varphi(g) \wedge \chi(h)$ for every $g \in V$ and $h \in E$, then $\psi$ is named as fuzzy incidence (FI) of $G$, and $(\varphi, \chi)$ is known as the fuzzy subgraph of $G$; if $\psi$ is FI of $G$, then $G=(\varphi, \chi, \psi)$ is known as a FIG of $G$.

Remark 1 (see [36]). If $\varphi(u)>0$, then $u$ is in the support of $\varphi$ where $u \in V$. If $\chi(\mathrm{uv})>0$, then uv is in the support of $\chi$ 


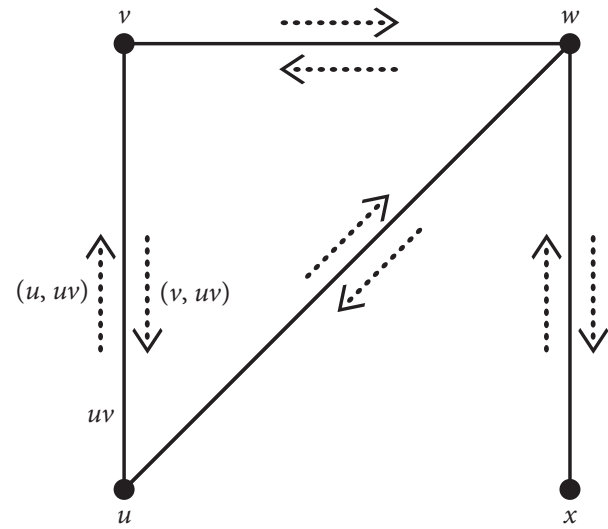

Figure 1: A FIG.

where uv $\in V \times V$, and if $\psi(u, \mathrm{uv})>0$, then (u, uv) is in the support of $\psi$ where $(u, \mathrm{uv}) \in V \times E . \varphi^{*}, \chi^{*}$, and $\psi^{*}$ are representing supports of $\varphi, \chi$, and $\psi$, respectively.

If the value of an IP $\psi(u, \mathrm{uv})$ or $\psi(v, \mathrm{vu})$ is not given in the FIG, then its value will be equal to zero. Also, two vertices $u$ and $v$ are connected in the FIG if there exists a path such that $u,(u, \mathrm{uv})$, uv, $(v, \mathrm{uv}), v$ between $u$ and $v$.

Definition 3 (see [36]). A FIG is said to be CFIG if $\psi(i, \mathrm{ij})=$ $\varphi(i) \wedge \chi(\mathrm{ij})$ for each $\psi(i, \mathrm{ij}) \in \psi^{*}$. Also, $\psi(i, \mathrm{ij})=\psi(j$, ji $)$ for each $i, j \in \varphi^{*}$. It is denoted by $K^{*}$.

Definition 4 (see [39]). Let $G$ be a FIG; the incidence degree $\left(d_{i}\right)$ of a node $u \in \varphi^{*}$ is defined as $d_{i}(u)=\sum_{u \neq v} \psi(u, u v)$.

The lowest $d_{i}$ of $G$ is defined by $\Omega(G)=\min \left\{d_{i}(v) \mid v \in V\right\}$.

The highest $d_{i}$ of $G$ is defined by $\Delta(G)=\max \left\{d_{i} \mid v \in V\right\}$.

\section{Relationship between the Order and Size of Fuzzy Incidence Graphs}

In this section, we define the order and size of the FIG. We also discuss a relationship between the order and the size of the FIG.

Definition 5. Assume $G=(\varphi, \chi, \psi)$ is a FIG. Then, $O(G)=$ $\sum_{u \neq v, u, v \in V} \psi(u$, uv $)$ is called the order of $G$, and $S(G)=\sum_{e \in \chi^{*}} \chi(e)$ is called the size of $G$.

Example 1. Suppose three garment factories are working with each other on the basis of some pros and cons. The MSV of the vertices represents the number of workers working in a factory, the MSV of an edge indicates the contract policies of these factories with each other, and the MSV of an IP shows the annual profit of a factory. We want to calculate the total annual profit of these factories. This can be done by using the definition of $O(G)$ of FIGs.

Assume $G=(\varphi, \chi, \psi)$ is a FIG having $\varphi=\{x, y, z\}$; $\varphi(x)=0.5, \varphi(y)=0.6, \varphi(z)=0.9 ; \chi(\mathrm{xy})=0.5, \chi(\mathrm{xz})=0.4$, $\chi(\mathrm{yz})=0.5 ; \psi \quad(x, \mathrm{xy})=0.4, \psi(y, \mathrm{yx})=0.3, \psi(x, \mathrm{xz})=0.3$, $\psi(z, \mathrm{zx})=0.4, \quad \psi(y, \mathrm{yz})=0.5$, and $\psi(z, \mathrm{zy})=0.4 . \quad$ Then, $O(G)=2.3$ is the total annual profit of these factories with $S(G)=1.4$.

Proposition 1. In a $F I G G=(\varphi, \chi, \psi), S(G) \leq O(G)$.

Proof. Let $G=(\varphi, \chi, \psi)$ be a FIG with one vertex. Then, $O(G)=S(G)=0$, i.e.,

$$
O(G)=S(G) .
$$

It is a trivial case. Assume $G$ with more than one vertex. $O(G)$ is the sum of all IPs of $G$. Since IPs are 2 times of edges, the total sum of all the MSVs of the IPs will always be greater than the total sum of all MSVs of the edges.

$$
S(G)<O(G) \text {. }
$$

From equations (1) and (2), we get

$$
S(G) \leq O(G) .
$$

Proposition 2. For any FIG, the following inequality holds: $O(G) \geq S(G) \geq \Delta(G) \geq \Omega(G)$.

Proof. Assume $G=(\varphi, \chi, \psi)$ is a FIG with a nonempty vertex set. Since $\Omega(G)$ represents lowest $d_{i}$ and $\Delta(G)$ denotes highest $d_{i}$ of $G$,

$$
\Delta(G) \geq \Omega(G) .
$$

We know $O(G)=\sum_{u \neq v, u, v \in V} \psi(u$, uv $) \quad$ and $S(G)=\sum_{e \in \chi^{*} \chi}(e)$.

By the definition of the size of $G, S(G)=\sum_{e \in \chi^{*}} \chi$ $(e) \geq \vee\left\{d_{i}(v) \mid v \in V\right\}$, i.e.,

$$
\Delta(G) \leq S(G) .
$$

By Proposition 1,

$$
S(G) \leq O(G) .
$$

From inequalities (4)-(6), we obtained $O(G) \geq S$ $(G) \geq \Delta(G) \geq \Omega(G)$.

Mordeson has shown $\sum_{u \in \sigma^{*}}\left(d_{i}(u)\right) \leq 2 \sum_{e \in \mu^{*}} \mu(e)$ [39]. In his result, there is an inequality. We are going to propose this type of result with equality but in the form of IPs.

Proposition 3. The $d_{i}$ sum of all vertices in a FIG is equal to twice the average sum of all the IPs, i.e.,

$$
\sum_{v \in \varphi^{*}} d_{i}(v)=2 \sum_{u, v \in V}\left(\frac{\psi(u, \mathrm{uv})+\psi(v, \mathrm{vu})}{2}\right) .
$$

Proof. Let $G=(\varphi, \chi, \psi)$ be a FIG, where $V=\left\{v_{1}, v_{2}, v_{3}, \ldots, v_{n}\right\}, \varphi \subseteq V, \chi \subseteq E$, and $\psi \subseteq V \times E$. Since $d_{i}(v)=\sum_{u \neq v} \psi(u, \mathrm{uv})$, 


$$
\begin{gathered}
d_{i}\left(v_{1}\right)=\psi\left(v_{1}, v_{1} v_{2}\right)+\psi\left(v_{1}, v_{1} v_{3}\right)+\cdots+\psi\left(v_{1}, v_{1} v_{n}\right) \\
d_{i}\left(v_{2}\right)=\psi\left(v_{2}, v_{2} v_{1}\right)+\psi\left(v_{2}, v_{2} v_{3}\right)+\cdots+\psi\left(v_{2}, v_{2} v_{n}\right) \\
\vdots \\
d_{i}\left(v_{n}\right)=\psi\left(v_{n}, v_{n} v_{1}\right)+\psi\left(v_{n}, v_{n} v_{2}\right)+\cdots+\psi\left(v_{n}, v_{n} v_{n-1}\right) .
\end{gathered}
$$

This implies $\sum_{v \in V} d_{i}(v)=d_{i}\left(v_{1}\right)+d_{i}\left(v_{2}\right)+\cdots+d_{i}\left(v_{n}\right)$. $\sum_{v \in V} d_{i}(v)=\psi\left(v_{1}, v_{1} v_{2}\right)+\psi\left(v_{1}, v_{1} v_{3}\right)+\cdots+\psi\left(v_{1}, v_{1} v_{n}\right)$

$$
\begin{aligned}
& +\psi\left(v_{2}, v_{2} v_{1}\right)+\psi\left(v_{2}, v_{2} v_{3}\right) \\
& +\cdots+\psi\left(v_{2}, v_{2} v_{n}\right)+\cdots \\
& +\psi\left(v_{n}, v_{n} v_{1}\right)+\psi\left(v_{n}, v_{n} v_{2}\right)+\cdots+\psi\left(v_{n}, v_{n} v_{n-1}\right) \\
\sum_{v \in V} d_{i}(v)= & \frac{2}{2} \psi\left(v_{1}, v_{1} v_{2}\right)+\psi\left(v_{1}, v_{1} v_{3}\right)+\cdots+\psi\left(v_{1}, v_{1} v_{n}\right) \\
& +\psi\left(v_{2}, v_{2} v_{1}\right)+\psi\left(v_{2}, v_{2} v_{3}\right) \\
& +\cdots+\psi\left(v_{2}, v_{2} v_{n}\right)+\cdots \\
& +\psi\left(v_{n}, v_{n} v_{1}\right)+\psi\left(v_{n}, v_{n} v_{2}\right)+\cdots+\psi\left(v_{n}, v_{n} v_{n-1}\right) .
\end{aligned}
$$

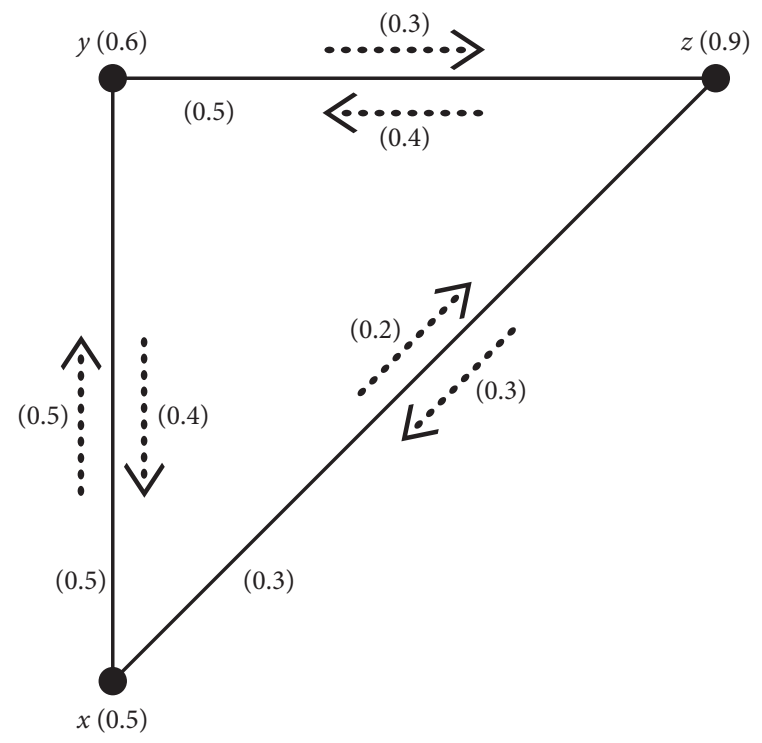

FIGURE 2: A FIG with $\sum d_{i}\left(v_{i}\right)=2.1=2 \sum_{u, v \in V}(\psi(u, u v) / 2)=$ 2(1.05).

By rearranging the terms,

$$
\begin{aligned}
\sum_{v \in V} d_{i}(v)= & 2\left(\frac{\psi\left(v_{1}, v_{1} v_{2}\right)+\psi\left(v_{2}, v_{2} v_{1}\right)}{2}+\frac{\psi\left(v_{1}, v_{1} v_{3}\right)+\psi\left(v_{3}, v_{3} v_{1}\right)}{2}\right. \\
& +\cdots+\frac{\psi\left(v_{1}, v_{1} v_{n}\right)+\psi\left(v_{n}, v_{n} v_{1}\right)}{2}+\frac{\psi\left(v_{2}, v_{2} v_{3}\right)+\psi\left(v_{3}, v_{3} v_{2}\right)}{2}+\frac{\psi\left(v_{2}, v_{2} v_{4}\right)+\psi\left(v_{4}, v_{4} v_{2}\right)}{2} \\
& \left.+\cdots+\frac{\psi\left(v_{2}, v_{2} v_{n}\right)+\psi\left(v_{n}, v_{n} v_{2}\right)}{2}+\cdots+\frac{\psi\left(v_{n-1}, v_{n-1} v_{n}\right)+\psi\left(v_{n}, v_{n} v_{n-1}\right)}{2}\right), \\
\sum_{v \in V} d_{i}(v)= & 2 \sum_{u, v \in V}\left(\frac{\psi(u, \mathrm{uv})+\psi(v, \mathrm{vu})}{2}\right) .
\end{aligned}
$$

Example 2. A FIG $G=(\varphi, \chi, \psi)$ is provided in Figure 2 with $\varphi=\{x, y, z\}$. We have $\sum d_{i}\left(v_{i}\right)=2.1$ and $\sum_{u, v \in V}(\psi(u, \mathrm{uv})+\psi(v, \mathrm{vu}) / 2)=1.05$. This implies $\sum d_{i}\left(v_{i}\right)=2 \sum_{u, v \in V}(\psi(u, \mathrm{uv})+\psi(v, \mathrm{vu}) / 2)$.

\section{Domination in Fuzzy Incidence Graphs}

Domination in graphs has plenty of uses in various fields. In this section, we have introduced the concepts of effective $d_{i}$, minimum effective $d_{i}$, maximum effective $d_{i}$, neighborhood incidence degree $\left(N d_{i}\right)$, minimum $N d_{i}$, maximum $N d_{i}$, FIDS, and FIDN for FIGs. The main benefit of our work is that if we know effective $d_{i}$ and $N d_{i}$ of the FIG, then we can study the further properties of the FIG. Also, in future, with the help of these ideas, we will be able to find effective $d_{i}$, minimum effective $d_{i}$, maximum effective $d_{i}, N d_{i}$, minimum $N d_{i}$, maximum $N d_{i}$, FIDS, and FIDN in union, join, composition, normal product, tensor product, and Cartesian product of FIGs.

Definition 6. An IP of a FIG is named as an effective incidence pair (EIP) if $\psi(i, \mathrm{ij})=\varphi(i) \wedge \chi(\mathrm{ij})$ for all $i \in V$, ij $\in E$.

Definition 7. Open incidence neighborhood (IN) is defined as $\operatorname{IN}(i)=\{j \in V \mid \psi(i, \mathrm{ij})=\varphi(i) \wedge \chi(\mathrm{ij})\}$. Closed incidence neighborhood of $i$ is $\operatorname{FIN}[i]=\operatorname{FIN}(i) \cup\{i\}$.

For a FIG, $d_{i}$ of a node can be generalized in distinct ways. 
Definition 8. The effective $d_{i}$ of a node $m$ is described as $\operatorname{dEIP}(m)=\sum \psi(m, \mathrm{mn})$. The minimum effective $d_{i}$ is denoted by $\delta_{\mathrm{dEIP}}(G)=\min \{\operatorname{dEIP}(m) \mid m \in V\}$. The maximum effective $d_{i}$ is denoted by $\Delta_{\mathrm{dEIP}}(G)=\max \{\operatorname{dEIP}(m) \mid m \in V\}$.

Definition 9. $N d_{i}$ of a node $m$ is expressed as $N d_{i}(m)=\sum_{n \in \operatorname{IN}(m)} \varphi(n)$. The minimum $N d_{i}$ is defined by $\delta_{\mathrm{dIN}}(G)=\min \{\operatorname{dIN}(m) \mid m \in V\}$. The maximum $N d_{i}$ is defined by $\Delta_{\mathrm{dIN}}(G)=\max \{\operatorname{dIN}(m) \mid m \in V\}$.

Definition 10. A vertex $i$ in a FIG $G=(\varphi, \chi, \psi)$ dominates vertex $j$ if $\psi(i, \mathrm{ij})=\varphi(i) \wedge \chi(\mathrm{ij})$, and a vertex $j$ dominates $i$ if $\psi(j, \mathrm{ij})=\varphi(j) \wedge \chi(\mathrm{ij})$. The set of these types of vertices is called a FIDS of the FIG.

Definition 11. A FIDS $D$ is called a minimal FIDS of $G$ if no proper subset of $D$ is a FIDS of $G$.

Definition 12. The FIDN is the minimum fuzzy cardinality (FC) of the FIDS among all FIDSs in G. It is represented by $\gamma_{\mathrm{FI}}$.

Example 3. Let us look at a practical example involving a FIDS and FIDN. As an illustrative case, consider a FIG in which vertices show different cities and the MSV of the vertices indicates the population of the cities. The MSV of the edges shows the mutual understanding of all these cities with each other, and the MSV of the IPs represents the traffic flow from one city to another city. FIDS will help us to find which city/cities has the maximum traffic flow to all other cities.

A FIG is shown in Figure 3 with

$$
\begin{aligned}
\varphi & =\{w, x, y, z\}, \\
\varphi(w) & =0.5, \\
\varphi(x) & =0.3, \\
\varphi(y) & =0.5, \\
\varphi(z) & =1, \\
\chi(\mathrm{wx}) & =0.3, \\
\chi(\mathrm{wy}) & =0.5, \\
\chi(\mathrm{xy}) & =0.2, \\
\chi(\mathrm{yz}) & =0.5, \\
\psi(w, \mathrm{wx}) & =0.3, \\
\psi(w, \mathrm{wy}) & =0.5, \\
\psi(x, \mathrm{xw}) & =0.2, \\
\psi(x, \mathrm{xy}) & =0.2, \\
\psi(y, \mathrm{yw}) & =0.5, \\
\psi(y, \mathrm{yx}) & =0.2, \\
\psi(y, \mathrm{yz}) & =0.5, \\
\psi(z, \mathrm{zy}) & =0.4 .
\end{aligned}
$$

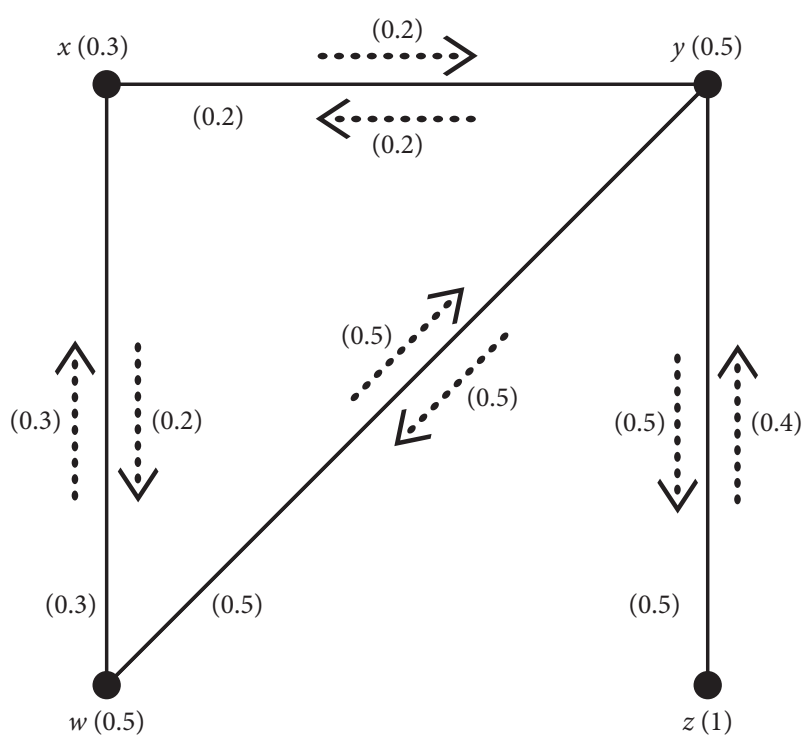

Figure 3: FIG with $\gamma_{\mathrm{FI}}=0.5$.

The minimal FIDS is $Q=\{y\}$, and FIDN $=\gamma_{\mathrm{FI}}=\varphi(y)=0.5$. This shows $y$ is the city through which the flow of traffic to all other cities is maximum.

\section{Remark 2}

(1) For any $u, v \in V$, if $u$ dominates $v$, then it is not necessary that $v$ dominates $u$.

(2) If $\psi(u, \mathrm{uv})<\varphi(u) \wedge \chi$ (uv), $\forall u \in V$, uv $\in E$. This implies $V$ is the unique FIDS of $G$. Conversely, if $V$ is the only FIDS of $G$, then $\psi(u, \mathrm{uv})<\varphi(u) \wedge \chi(\mathrm{uv}), \forall u \in V$, uv $\in E$.

(3) For the CFIG, $\{i\}$ is a FIDS for every $i$ belonging to $V$, and we have $\gamma_{\mathrm{FI}}\left(K^{*}\right)=\min _{x \in V} \varphi(x)$.

Definition 13. A node $m$ of FIG $G=(\varphi, \chi, \psi)$ is named as an isolated node if $\psi(m, \mathrm{mn})<\varphi(m) \wedge \chi(\mathrm{mn}), \forall n \in V-\{m\}$, i.e., $\operatorname{FIN}(m)=\varnothing$. Therefore, in the FIG, no node is dominated by an isolated node, but an isolated node dominates itself.

Definition 14. Assume $G=(\varphi, \chi, \psi)$ is a FIG; the complement of $G=(\varphi, \chi, \psi)$ is a FIG which is represented by $\bar{G}=$ $(\bar{\varphi}, \bar{\chi}, \bar{\psi})$ and is defined as

$$
\begin{aligned}
& \text { (i) } \bar{\varphi}(a)=\varphi(a) \\
& \text { (ii) } \bar{\chi}(\mathrm{ab})=\varphi(a) \wedge \varphi(b)-\chi(\mathrm{ab}) \\
& \text { (iii) } \bar{\psi}(a, \mathrm{ab})=\min (\max (\varphi(a), \chi(\mathrm{ab}))- \\
& \psi(a, \mathrm{ab}), \varphi(a) \wedge \varphi(b)-\chi(\mathrm{ab}))
\end{aligned}
$$

Example 4. Assume $G=(\varphi, \chi, \psi)$ is a FIG having $\varphi=\{w, x, y, z\}$

$\varphi(w)=0.3, \varphi(x)=0.5, \varphi(y)=0.9, \varphi(z)=0.7$;

$\chi(\mathrm{wx})=0.3, \chi(\mathrm{wy})=0.2, \chi(\mathrm{xy})=0.4, \chi(\mathrm{yz})=0.6 ; \quad$ and $\psi(w, \mathrm{wx})=0.3, \psi(x, \mathrm{xw})=0.3, \psi(x, \mathrm{xy})=0.3, \psi$

$(y, \mathrm{yx})=0.2, \psi(w, \mathrm{wy})=0.1, \psi(y, \mathrm{yw})=0.1$,

$\psi(y, \mathrm{yz})=0.5, \psi(z, \mathrm{zy})=0.5$. 
Its complement $\bar{G}$ is shown in Figure 4 .

Theorem 1. For any FIG, $G=(\varphi, \chi, \psi) 2 p>\gamma_{F I}+\bar{\gamma}_{F I}$, where $\gamma_{F I}$ and $\bar{\gamma}_{F I}$ are the FIDN of $G$ and $\bar{G}$, respectively.

\section{Strong and Weak Domination in Fuzzy Incidence Graphs}

In this section, we have discussed strong and weak FID for FIGs and give different examples to understand these concepts. The results provided in this section are based on $[19,40]$. In this view, similar results related to the strong FIDN and weak FIDN in FIGs are achieved.

Definition 15. Assume $G=(\varphi, \chi, \psi)$ is a FIG, and let $i$ and $j$ be the nodes of $G$. Then, $i$ strongly dominates $j$ or $j$ weakly dominates $i$ if the following two conditions are satisfied:
(i) $d_{i}(i) \geq d_{i}(j)$
(ii) $\psi(i, \mathrm{ij})=\varphi(i) \wedge \chi(\mathrm{ij})$

We call $j$ strongly dominates $i$ or $i$ weakly dominates $j$ if

(i) $d_{i}(j) \geq d_{i}(i)$

(ii) $\psi(j, j i)=\varphi(j) \wedge \chi($ ji $)$

Definition 16. A set $R \subseteq V$ is a strong FIDS if each node in $V-R$ is strongly fuzzy incidence dominated by at least one node in $R$. In a similar way, $R$ is called a weak FIDS if each node in $V-R$ is weakly fuzzy incidence dominated by at least one node in $R$.

Definition 17. The lowest FC of a strong FIDS is uttered as the strong FIDN, and it is represented by $\gamma_{\mathrm{SFI}}(G)$ or $\gamma_{\mathrm{SFI}}$, and the lowest FC of a weak FIDS is named as the weak FIDN, and it is represented by $\gamma_{\mathrm{WFI}}(G)$ or $\gamma_{\mathrm{WFI}}$.

Example 5. Assume $G=(\varphi, \chi, \psi)$ is a FIG as shown in Figure 5 having $\varphi=\{w, x, y, z\} ; \varphi(w)=1, \varphi(x)=0.7$, $\varphi(y)=0.5, \varphi(z)=1, \chi(\mathrm{wx})=0.6, \chi(\mathrm{wy})=0.4, \chi(\mathrm{xy})=0.5$, $\chi(\mathrm{yz})=0.4, \psi(w, \mathrm{wx})=0.5, \psi(x, \mathrm{xw})=0.6, \psi(x, \mathrm{xy})=0.4$, $\psi(y, \mathrm{yx})=0.5, \psi(w, \mathrm{wy})=0.4, \psi \quad(y, \mathrm{yw})=0.4, \psi(y, \mathrm{yz})$ $=0.4$, and $\psi(z, \mathrm{zy})=0.4$. Assume $R=\{y\}$. We have $V-R=\{w, x, z\}$. Here, $y$ strongly fuzzy incidence dominates $w, x$, and $z$ because $d_{i}(y)=1.3$ is greater than $d_{i}$ of all the remaining vertices, i.e., $d_{i}(w)=1.0, d_{i}(x)=1.0$, and $d_{i}(z)=0.4$. There are no other strong FIDSs. Thus, the only strong FIDS is $R=\{y\}$. Therefore, $\gamma_{\text {SFI }}=0.5$. We have a weak FIDS which is $R_{1}=\{w, x, z\}$ with $\gamma_{\mathrm{WFI}}=\varphi(w)+$ $\varphi(x)+\varphi(z)=1+0.7+1=2.7$.

Remark 3. If $G$ is not a CFIG, then $\gamma_{\mathrm{SFI}}<\gamma_{\mathrm{WFI}}$.

Theorem 2. For any CFIG with $\psi(i, \mathrm{ij})=\varphi(i) \wedge \chi(\mathrm{ij})$ for all $i \in V$ and $\mathrm{ij} \in E$, the inequality given in the following always holds:

$$
\gamma_{\mathrm{WFI}} \leq \gamma_{\mathrm{SFI}}
$$
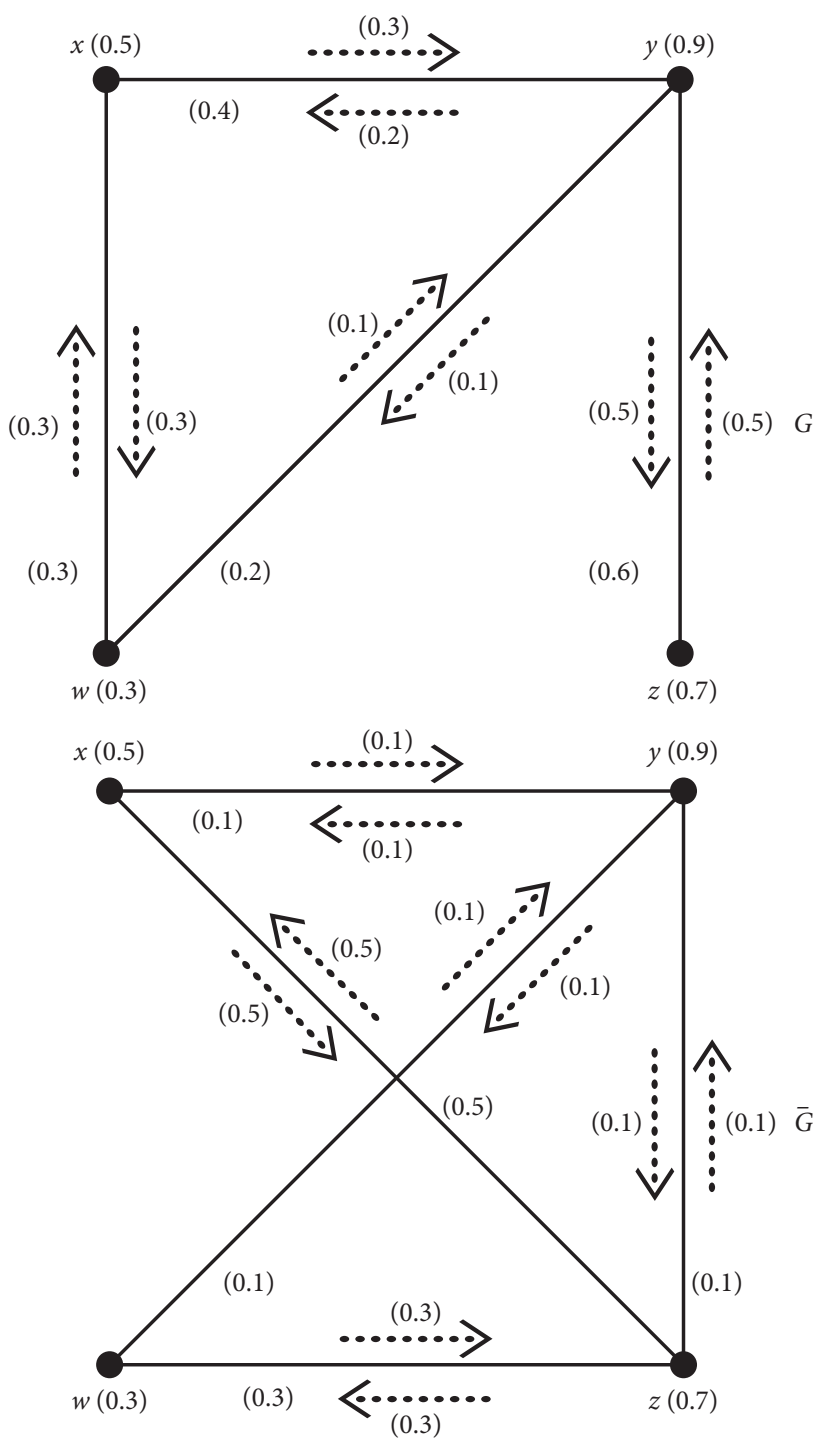

Figure 4: Graph $G$ is complement to $\bar{G}$.

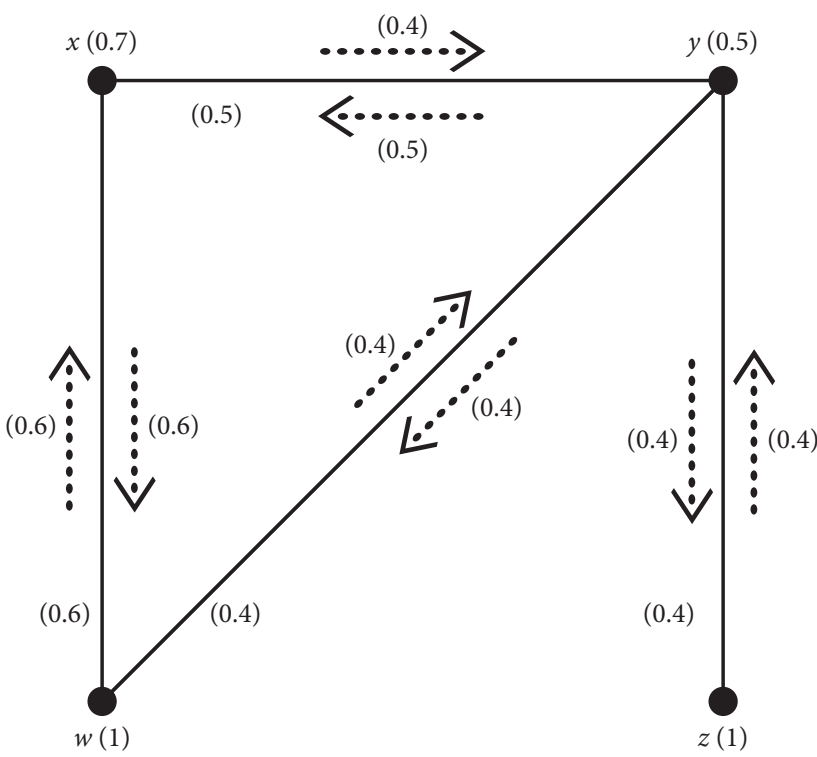

FIGURE 5: $G$ having $\gamma_{\mathrm{SFI}}<\gamma_{\mathrm{WFI}}$. 
Proof. Let $G=(\varphi, \chi, \psi)$ be a CFIG with $\psi(i, \mathrm{ij})=\varphi(i) \wedge \chi(\mathrm{ij})$. Assume for every $w_{i} \in V, \varphi\left(w_{i}\right)$ are the same. Since $G$ is a CFIG with $\chi\left(w_{i} w_{j}\right)=\varphi\left(w_{i}\right) \wedge \varphi\left(w_{j}\right)$ for all $w_{i}, w_{j} \in V$ and $\psi\left(w_{i}, w_{i} w_{j}\right)=\varphi\left(w_{i}\right) \wedge \chi\left(w_{i} w_{j}\right)$ for all $w_{i} \in V, w_{i} w_{j} \in E$.

Thus, every $w_{i} \in V$ is a strong as well as weak FIDS; therefore,

$$
\gamma_{\mathrm{SFI}}=\gamma_{\mathrm{WFI}}
$$

Assume for all $w_{i} \in V, \varphi\left(w_{i}\right)$ are not the same. In a CFIG with $d_{i}\left(w_{i}\right) \geq d_{i}\left(w_{j}\right)$, from all the nodes, one of them strongly dominates all the remaining nodes; if it is smallest among all the nodes, then the FIDS with that node is called a weak FIDN that is $\gamma_{\mathrm{WFI}}=\varphi\left(w_{i}\right)$ with $d_{i}\left(w_{i}\right) \leq d_{i}\left(w_{j}\right)$ for all $w_{i}, w_{j} \in V$ and $\psi\left(w_{i}, w_{\mathrm{ij}}\right)=\varphi\left(w_{i}\right) \wedge \chi\left(w_{i} w_{j}\right)$ for all $w_{i} \in V$, $w_{i} w_{j} \in E$.

Certainly, the strong FIDS has a node set other than that node set. This implies

$$
\gamma_{\mathrm{WFI}}<\gamma_{\mathrm{SFI}}
$$

and from equations (13) and (14), we get $\gamma_{\mathrm{WFI}} \leq \gamma_{\mathrm{SFI}}$.

Example 6. Assume $G=(\varphi, \chi, \psi)$ is a CFIG having $\varphi=\{x, y, z\} ; \quad \varphi(x)=0.5, \varphi(y)=0.3, \varphi(z)=0.8 ; \quad \chi(\mathrm{xy})=$ $0.3, \chi(\mathrm{xz})=0.5, \chi(\mathrm{yz})=0.3$; and $\psi(x, \mathrm{xy})=0.3, \psi(y, \mathrm{yx})=$ $0.3, \psi(y, \mathrm{yz})=0.3, \psi(z, \mathrm{zy})=0.3, \psi(x, \mathrm{xz})=0.5, \psi \quad(z, \mathrm{zx})$ $=0.5$. Here, $D_{1}=\{x\}$ is a strong FIDS which strongly dominates $\{y, z\}$ and $D_{2}=\{z\}$ is another strong FIDS because it also strongly dominates $\{x, y\}$. Therefore, $\gamma_{\mathrm{SFI}}=0.5$ and $\gamma_{\mathrm{WFI}}=0.3$.

Theorem 3. For a CFIG, the inequalities given in the following are true:

(i) $\gamma_{F I} \leq \gamma_{S F I} \leq O(G)$-highest $d_{i}$ of $G$

(ii) $\gamma_{F I} \leq \gamma_{W F I} \leq O(G)$-lowest $d_{i}$ of $G$

\section{Proof}

(i) From Definitions 15-17, we have

$$
\gamma_{\mathrm{FI}} \leq \gamma_{\mathrm{SFI}}
$$

We know $O(G)=p$, the sum of $d_{i}$ of the FIG. Also,

$$
O(G) \text { - not including the highest } d_{i} \text { of the FIG }=O(G)-\Delta(G) \text {. }
$$

From equations (15) and (16), $\gamma_{\mathrm{FI}} \leq \gamma_{\mathrm{SFI}} \leq \mathrm{O}$ (G)-highest $d_{i}$ of $G$.

(ii) From Definitions 15-17, the weight of $\gamma_{\mathrm{FI}}$ of the FIG is less than or equal to $\gamma_{\mathrm{WFI}}$ of the FIG as elements of $F$ weakly dominate any one of elements of $V-F$. Therefore, the weak FIDN will be greater than or equal to $\gamma_{\mathrm{FI}}$.

$$
\gamma_{\mathrm{WFI}}(G) \geq \gamma_{\mathrm{FI}}(G)
$$

Also,

$$
O(G)-\delta(G)=p-\delta(G)
$$

From equations (17) and (18), we get $\gamma_{\mathrm{FI}} \leq \gamma_{\mathrm{WFI}} \leq O(G)$-lowest $d_{i}$ of $G$.

Example 7. Assume $G=(\varphi, \chi, \psi)$ is a CFIG having $\varphi=\{x, y, z\} ; \quad \varphi(x)=0.8, \varphi(y)=0.3, \varphi(z)=0.9 ; \quad \chi(\mathrm{xy})$ $=0.3, \chi(\mathrm{xz})=0.8, \chi(\mathrm{yz})=0.3 ; \quad$ and $\quad \psi(x, \mathrm{xy})=0.3, \psi$ $(y, \mathrm{yx})=0.3, \psi(y, \mathrm{yz})=0.3, \psi(z, \mathrm{zy})=0.3, \psi(x, \mathrm{xz})=0.8, \psi$ $(z, \mathrm{zx})=0.8 . \quad d_{i}(x)=1.1, d_{i}(y)=0.6, d_{i}(z)=1.1, \gamma_{\mathrm{FI}}=$ $0.3, \gamma_{\mathrm{SFI}}=0.8, \gamma_{\mathrm{WFI}}=0.3$, order of $G=2.8$, highest $d_{i}$ of $G=1.1$, and lowest $d_{i}$ of $G=0.6$. Hence, Theorem 3 can be verified.

\section{Algorithm and Application of FID in the Selection of the Best Medical Lab}

In this section, an algorithm to find the FIDS and FIDN for the FIG is provided. We apply our algorithm in the real-life example to select the best medical lab among different labs (Algorithm 1).

6.1. Algorithm: Steps to Find the FIDS and FIDN. 6.2. Application to Select the Best Medical Lab. Suppose there are six different medical labs which are working in a city for conducting tests of coronavirus. Here, in our study, we are not mentioning the original names of these labs; therefore, consider the labs $l_{1}, l_{2}, l_{3}, l_{4}, l_{5}$, and $l_{6}$. In FIGs, the vertices show the labs, and edges show the contract conditions among the labs to share the facilities or test kits. The IPs show the transferring of patients from one lab to another lab due to the lack of resources (machinery, equipment, kits, and doctors). The FIDS of the FIG is the set of labs which perform the tests independently. In this way, we can save the time of patients and overcome the long traveling of patients by providing the few facilities to these labs.

Assume $G=(\varphi, \chi, \psi)$ is a FIG shown in Figure 6 having 
Step 1: input the vertex set $V$, edge set $E \subseteq V \times V$, and IP set $I \subseteq V \times E$.

Step 2: sort $V$ such that $V=\left\{\varphi\left(l_{i}\right) \leq \varphi\left(l_{i+1}\right) \leq \cdots \leq\left(l_{n}\right)\right\}$.

Step 3: LOOP for $j=1$ to the number of vertices

Let $\mathrm{FIDS}_{j}=\varnothing, \mathrm{NDS}=\varnothing, W=\varnothing$, and $\operatorname{Sum}=\varnothing$;

Put $i=j$

Step 4: LOOP For $v_{i} \in V$

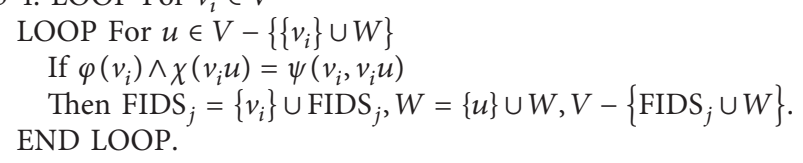

If $v_{i} \in$ FIDS $_{j}$

Then go to Step 4.

Else NDS $=\left\{v_{i}\right\} \cup$ NDS.

END LOOP If $(V=\varnothing)$.

Step 5: LOOP For $v_{i} \in W$

LOOP For $u \in$ NDS

If $\varphi\left(v_{i}\right) \wedge \chi\left(v_{i} u\right)=\psi\left(v_{i}, v_{i} u\right)$

Then FIDS $_{j}=$ FIDS $_{j} \cup\left\{v_{i}\right\}$ and NDS $=$ NDS $-\{u\}$

$$
\text { END LOOP }
$$

END LOOP

Step 6: If NDS $\neq \varnothing$ Then FIDS $_{j}=$ FIDS $_{j} \cup$ NDS.

Step 7: Sum $=\left[\operatorname{Sum~Sum~}\left(\right.\right.$ FIDS $\left.\left._{j}\right)\right]$;

$j=j+1$

If $j \leq$ number of vertices

Then Go To Step 3

Else

END LOOP (Step 3)

Step 8: FIDN is the minimum value of array Sum.

Algorithm 1: Steps to find the FIDS and FIDN.

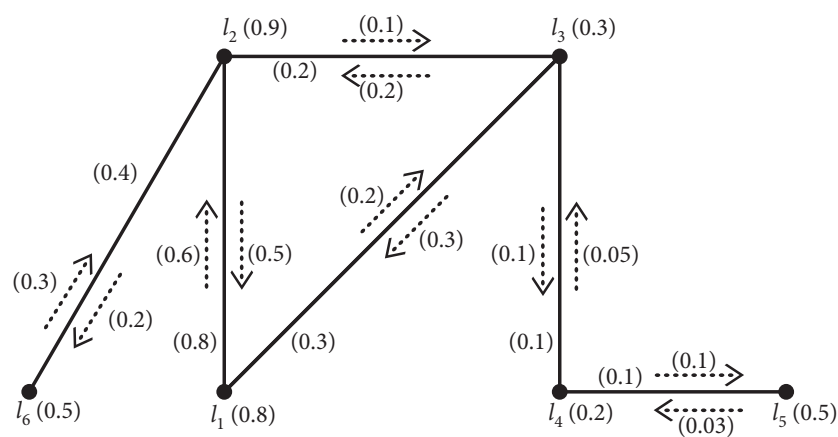

FIgURe 6: A FIG with $\gamma \mathrm{FI}=1$. 
Step 1: $V=\left\{\varphi\left(l_{1}\right)=0.8, \varphi\left(l_{2}\right)=0.9, \varphi\left(l_{3}\right)=0.3, \varphi\left(l_{4}\right)=0.2, \varphi\left(l_{5}\right)=0.5, \varphi\left(l_{6}\right)=0.5\right\}, E=\left\{\chi\left(l_{1} l_{2}\right)=0.8, \chi\left(l_{1} l_{3}\right)=0.3, \chi\left(l_{2} l_{3}\right)=0.2\right.$, $\left.\chi\left(l_{2} l_{6}\right)=0.4, \chi\left(l_{3} l_{4}\right)=0.1, \chi\left(l_{4} l_{5}\right)=0.1\right\}$, and $I=\left\{\psi\left(l_{1}, l_{1} l_{2}\right)=0.6, \psi\left(l_{2}, l_{1} l_{2}\right)=0.5, \psi\left(l_{1}, l_{1} l_{3}\right)=0.2, \psi\left(l_{3}, l_{1} l_{3}\right)=0.3, \psi\left(l_{2}, l_{2} l_{3}\right)=\right.$ $\left.0.1, \psi\left(l_{3}, l_{2} l_{3}\right)=0.2, \psi\left(l_{2}, l_{2} l_{6}\right)=0.2, \psi\left(l_{6}, l_{2} l_{6}\right)=0.3, \psi\left(l_{3}, l_{3} l_{4}\right)=0.1, \psi\left(l_{4}, l_{3} l_{4}\right)=0.05, \psi\left(l_{4}, l_{4} l_{5}\right)=0.1, \psi\left(l_{5}, l_{4} l_{5}\right)=0.6\right\}$.

Step 2: $V=\left\{\varphi\left(l_{4}\right)=0.2, \varphi\left(l_{3}\right)=0.3, \varphi\left(l_{5}\right)=0.5, \varphi\left(l_{6}\right)=0.5, \varphi\left(l_{1}\right)=0.8, \varphi\left(l_{2}\right)=0.9\right\}$.

Step 3: for $j=1 \mathrm{FIDS}_{1}=\varnothing$, NDS $=\varnothing, W=\varnothing$, and Sum $=\varnothing$.

put $i=1$

Step 4: $v_{1}=\varphi\left(l_{4}\right), l_{4}$ dominates $l_{5}$, and $l_{3}$ dominates $l_{1}, l_{2}$. Therefore, FIDS ${ }_{1}=\left\{l_{3}, l_{4}\right\}, W=\left\{l_{5}, l_{1}, l_{2}\right\}$, and NDS $=\left\{l_{6}\right\}$.

Step 5: for $\left\{l_{5}, l_{1}, l_{2}\right\} \in W$ dominates $\left\{l_{6}\right\} \in N D S$. This implies FIDS $_{1}=\left\{l_{3}, l_{4},\right\}$.

Step 6: since NDS $\neq \varnothing$, therefore, FIDS $_{1}=\left\{l_{3}, l_{4}, l_{6}\right\}$.

Step 7: Sum $=\left[\varphi\left(l_{3}\right)+\varphi\left(l_{4}\right)+\varphi\left(l_{6}\right)=0.3+0.2+0.5=1,1,1,1,1,1\right]$

For $j=2,3,4,5,6$

FIDS $=\left\{l_{3}, l_{4}, l_{6}\right\}$

For $j=7$

As $j>$ number of vertices

END LOOP (Step 3)

Step 8: FIDN $=1$.

Algorithm 2: Application of Algorithm 1.

$$
\begin{aligned}
\varphi & =\left\{l_{1}, l_{2}, l_{3}, l_{4}, l_{5}, l_{6}\right\} \\
\varphi\left(l_{1}\right) & =0.8 \\
\varphi\left(l_{2}\right) & =0.9 \\
\varphi\left(l_{3}\right) & =0.3 \\
\varphi\left(l_{4}\right) & =0.2, \\
\varphi\left(l_{5}\right) & =0.5 \\
\varphi\left(l_{6}\right) & =0.5 \\
\chi\left(l_{1} l_{2}\right) & =0.8 \\
\chi\left(l_{1} l_{3}\right) & =0.3 \\
\chi\left(l_{2} l_{3}\right) & =0.2 \\
\chi\left(l_{3} l_{4}\right) & =0.1 \\
\chi\left(l_{2} l_{6}\right) & =0.4 \\
\chi\left(l_{4} l_{5}\right) & =0.1 \\
\psi\left(l_{1}, l_{1} l_{2}\right) & =0.6 \\
\psi\left(l_{2}, l_{2} l_{1}\right) & =0.5 \\
\psi\left(l_{1}, l_{1} l_{3}\right) & =0.2 \\
\psi\left(l_{3}, l_{3} l_{1}\right) & =0.3 \\
\psi\left(l_{2}, l_{2} l_{3}\right) & =0.1 \\
\psi\left(l_{3}, l_{3} l_{2}\right) & =0.2 \\
\psi\left(l_{3}, l_{3} l_{4}\right) & =0.1 \\
\psi\left(l_{4}, l_{4} l_{3}\right) & =0.05 \\
\psi\left(l_{2}, l_{2} l_{6}\right) & =0.2 \\
\psi\left(l_{6}, l_{6} l_{2}\right) & =0.3 \\
\psi\left(l_{4}, l_{4} l_{5}\right) & =0.1 \\
\psi\left(l_{5}, l_{5} l_{4}\right) & =0.03
\end{aligned}
$$

Algorithm 2 is applied.
This shows that patients can visit any one of the labs from this set. The government should provide the resources to labs $l_{3}, l_{4}$, and $l_{6}$ for the proper and easy conduction of tests for coronavirus.

\section{Comparative Analysis}

In an FG, if vertex $u$ dominates vertex $v$, then vertex $u$ also dominates vertex $v$, but in FIGs, it is not necessary that if vertex $u$ dominates vertex $v$, then vertex $v$ also dominates vertex $u$. Therefore, FIGs are more interesting than FGs. Here, we are comparing our study with the previous study. In Figure 6, a FIG represents six different medical labs working with each other for conducting the coronavirus tests. We get FIDS $=\left\{l_{3}, l_{4}, l_{6}\right\}$ comprising three different medical labs which are best for the tests of coronavirus with $\gamma_{\mathrm{FI}}=1$. In Figure 6 , if we remove all the IPs, we get an FG. In the case of an FG, $l_{1}$ is dominating $l_{2}$ and $l_{3}$ because $\chi\left(l_{1} l_{2}\right)=0.8=\varphi\left(l_{1}\right) \wedge \varphi\left(l_{2}\right)=0.8 \wedge 0.9$ and $\chi\left(l_{1} l_{3}\right)=0.3=\varphi\left(l_{1}\right) \wedge \varphi\left(l_{3}\right)=0.8 \wedge 0.3$. The rest of the labs are not dominating each other. Therefore, DS $D=\left\{l_{1}, l_{4}, l_{5}, l_{6}\right\}$ with DN 2. In the case of an FG, the government needs to work on four labs, but in the case of the FIG, the government needs to work on three labs. So, the government should work on three labs instead of four labs. In this way, the government can save its money and time. This shows that our study is better than the previous studies.

\section{Conclusion}

The notion of domination in graphs is vital from a theoretical as well as an application point of view. Different authors have come out with more than thirty-five domination parameters. In this paper, we initiated novel ideas of FIGs, namely, order, size, a relationship between order and size, FIDS, strong FIDS, and weak FIDS. A relationship among order, FIDS, strong FIDS, and weak FIDS is also discussed. The formula to calculate $d_{i}$, effective $d_{i}$, and $N d_{i}$ 
of the FIG is explained. Our study will help us to find the order, size, FIDS, FIDN, $d_{i}$, effective $d_{i}$, and $N d_{i}$ for the union, join, composition, tensor product, normal product, and the Cartesian product of FIGs if we know the order, size, FIDS, FIDN, $d_{i}$, effective $d_{i}$, and $N d_{i}$ of two FIGs. The results explained in this paper may be used to study different FIG invariants. An algorithm to find the FIDS and FIDN is provided. An application of the FIDS for FIGs is provided to choose the best medical lab for conducting tests of coronavirus among different medical labs. Our objective is to extend our research work to Hamiltonian FIGs, bipolar FIGs, the coloring of FIGs, and the chromaticity of FIGs. Further work on these ideas will be reported in upcoming papers.

\section{Data Availability}

All the data used to support the findings of this study are included within the article.

\section{Conflicts of Interest}

The authors declare that there are no conflicts of interest regarding the publication of this paper.

\section{Acknowledgments}

This research work was partially supported by Ministerio de Ciencia, Innovacion y Universidades (Grant no. PGC2018097198-B-I00) and Fundación Séneca de la Region de Murcia (Grant no. 20783/PI/18).

\section{References}

[1] G. Chartrand and P. Zhang, "A first course in graph theory," Courier Corporation, vol. 3, 2013.

[2] S. M. Hosamani, V. B. Awati, and R. M. Honmore, "On graphs with equal dominating and c-dominating energy," Applied Mathematics and Nonlinear Sciences, vol. 4, no. 2, pp. 503512, 2019.

[3] P. S. R. Harisha, V. Lokesha, and S. Kumar, "Degree sequence of graph operator for some standard graphs," Applied Mathematics and Nonlinear Sciences, vol. 5, no. 2, pp. 99-108, 2020.

[4] B. Mathew, S. J. John, and H. Garg, Vertex Rough Graphs. Complex and Intelligent Systems, vol. 6, pp. 347-353, 2020.

[5] M. B. Belay and C. Wang, "The first general Zagreb coindex of graph operations," Applied Mathematics and Nonlinear Sciences, vol. 5, no. 2, pp. 109-120, 2020.

[6] S. Goyal, P. Garg, and V. N. Mishra, "New composition of graphs and their Wiener Indices," Applied Mathematics and Nonlinear Sciences, vol. 4, no. 1, pp. 163-168, 2019.

[7] B. M. Gurevich, "On classes of infinite loaded graphs with randomly deleted edges," Applied Mathematics and Nonlinear Sciences, vol. 5, no. 2, pp. 257-260, 2020.

[8] C. Natarajan and S. Ayyaswamy, "On strong (weak) domination in fuzzy graphs," International Journal of Mathematical and Computational Sciences, vol. 4, no. 7, pp. 1035-1037, 2010.

[9] A. Sahin, "Dichromatic polynomial for graph torus knot," Applied Mathematics and Nonlinear Sciences, pp. 1-6, 2020.
[10] M. Sunitha and A. Vijayakumar, "Complement of a fuzzy graph," Indian Journal of Pure and Applied Mathematics, vol. 33, no. 9, pp. 1451-1464, 2002.

[11] L. A. Zadeh, "Fuzzy sets," Information and Control, vol. 8, no. 3, pp. 338-353, 1965.

[12] A. Rosenfeld, Fuzzy Graphs. Fuzzy Sets and Their Applications to Cognitive and Decision Processes, pp. 77-95, Elsevier, Amsterdam, Netherlands, 1975.

[13] A. N. Gani and M. B. Ahamed, "Order and size in fuzzy graphs," Bulletin of Pure and Applied Sciences, vol. 22, no. 1, pp. $145-148,2003$.

[14] M. Akram, "Bipolar fuzzy graphs," Information Sciences, vol. 181, no. 24, pp. 5548-5564, 2011.

[15] P. Bhattacharya, "Some remarks on fuzzy graphs," Pattern Recognition Letters, vol. 6, no. 5, pp. 297-302, 1987.

[16] H. Rashmanlou and Y. B. Jun, "Complete interval-valued fuzzy graphs," Annals of Fuzzy Mathematics and Informatics, vol. 6, no. 3, pp. 677-687, 2013.

[17] A. Somasundaram and S. Somasundaram, "Domination in fuzzy graphs - I,” Pattern Recognition Letters, vol. 19, no. 9, pp. 787-791, 1998.

[18] A. Somasundaram, "Domination in products of fuzzy graphs," International Journal of Uncertainty, Fuzziness and Knowledge-Based Systems, vol. 13, no. 02, pp. 195-204, 2005.

[19] A. N. Gani and M. B. Ahamed, "Strong and weak domination in fuzzy graphs," East Asian Mathematical Journal, vol. 23, no. 1, pp. 1-8, 2007.

[20] O. Manjusha and M. Sunitha, "Total domination in fuzzy graphs using strong arcs," Annals of Pure and Applied Mathematics, vol. 9, no. 1, pp. 23-33, 2014.

[21] O. T. Manjusha and M. S. Sunitha, "Strong domination in fuzzy graphs," Fuzzy Information and Engineering, vol. 7, no. 3, pp. 369-377, 2015.

[22] C. Ponnappan, S. B. Ahamed, and P. Surulinathan, "Edge domination in fuzzy graphs new approach," International Journal of IT Engineering Applied Sciences Research, vol. 4, no. 1, pp. 14-17, 2015.

[23] K. Dharmalingam and M. Rani, "Equitable domination in fuzzy graphs," International Journal of Pure and Applied Mathematics, vol. 94, no. 5, pp. 661-667, 2014.

[24] K. Dharmalingam and P. Nithya, "Excellent domination fuzzy graphs," Bulletin of International Mathematical Virtual Institute7, vol. 7, no. 2, pp. 257-266, 2017.

[25] P. Nithya and K. Dharmalingam, "Very excellent domination in fuzzy graphs," International Journal of Computational and Applied Mathematics, vol. 12, no. 1, pp. 313-326, 2017.

[26] Y. Wang, C. K. Ahn, H. Yan, and S. Xie, "Fuzzy control and filtering for nonlinear singularly perturbed Markov jump systems," IEEE Transactions on Cybernetics, vol. 51, no. 1, pp. 297-308, 2020.

[27] Y. Wang, X. Xie, M. Chadli, S. Xie, and Y. Peng, "Sliding mode control of fuzzy singularly perturbed descriptor systems," IEEE Transactions on Fuzzy Systems, p. 1, 2020.

[28] K. R. Bhutani, J. Mordeson, and A. Rosenfeld, "On degrees of end nodes and cut nodes in fuzzy graphs," Iranian Journal of Fuzzy Systems, vol. 1, no. 1, pp. 57-64, 2004.

[29] A. N. Gani and K. P. Devi, "2-domination in fuzzy graphs," International Journal of Fuzzy Mathematical Archive, vol. 9, no. 1, pp. 119-124, 2015.

[30] A. N. Gani and A. A. Rahman, "Accurate 2-domination in fuzzy graphs using strong arcs," International Journal of Pure and Applied Mathematics, vol. 118, no. 6, pp. 279-286, 2018.

[31] L. Jiang, T. Zhang, and Y. Feng, "Identifying the critical factors of sustainable manufacturing using the fuzzy 
DEMATEL method," Applied Mathematics and Nonlinear Sciences, vol. 5, no. 2, pp. 391-404, 2020.

[32] O. T. Manjusha and M. S. Sunitha, "Notes on domination in fuzzy graphs," Journal of Intelligent \& Fuzzy Systems, vol. 27, no. 6, pp. 3205-3212, 2014.

[33] S. Mathew and M. S. Sunitha, "Types of arcs in a fuzzy graph," Information Sciences, vol. 179, no. 11, pp. 1760-1768, 2009.

[34] D. A. Xavior, F. Isido, and V. M. Chitra, "On domination in fuzzy graphs," International Journal of Computing Algorithm, vol. 2, pp. 248-250, 2013.

[35] T. Dinesh, "Fuzzy incidence graph - an introduction," Advances in Fuzzy Sets and Systems, vol. 21, no. 1, pp. 33-48, 2016.

[36] S. Mathew and J. N. Mordeson, "Connectivity concepts in fuzzy incidence graphs," Information Sciences, vol. 382-383, pp. 326-333, 2017.

[37] J. N. Mordeson and S. Mathew, "Fuzzy end nodes in fuzzy incidence graphs," New Mathematics and Natural Computation, vol. 13, no. 01, pp. 13-20, 2017.

[38] J. N. Mordeson, S. Mathew, and R. A. Borzooei, "Vulnerability and government response to human trafficking: vague fuzzy incidence graphs," New Mathematics and Natural Computation, vol. 14, no. 02, pp. 203-219, 2018.

[39] S. Mathew, J. N. Mordeson, and H. L. Yang, "Incidence cuts and connectivity in fuzzy incidence graphs," Iranian Journal of Fuzzy Systems, vol. 16, no. 2, pp. 31-43, 2019.

[40] J. N. Mordeson, S. Mathew, and D. S. Malik, "Fuzzy incidence graphs," Fuzzy Graph Theory with Applications to Human Trafficking, vol. 365, pp. 87-137, 2018. 